\title{
Preoperative MR Imaging to Differentiate Chordoid Meningiomas from Other Meningioma Histologic Subtypes
}

\author{
(D).D. Baal, (DW.C. Chen, (DD.A. Solomon, (D).S. Pai, (D) C.-H. Lucas, DJ.H. Hara, DN.A. Oberheim Bush, DM.W. McDermott,
} (DD.R. Raleigh, and (DJ.E. Villanueva-Meyer

\begin{abstract}
BACKGROUND AND PURPOSE: Chordoid meningiomas are uncommon WHO grade II primary intracranial neoplasms that possess unique chordoid histology and follow an aggressive clinical course. Our aim was to assess the utility of qualitative MR imaging features and quantitative apparent diffusion coefficient values as distinguishing preoperative MR imaging metrics to identify and differentiate chordoid histology from other meningioma histologic subtypes.
\end{abstract}

MATERIALS AND METHODS: Twenty-one patients with meningiomas with chordoid histology, which included both chordoid meningiomas ( $>50 \%$ chordoid histology) and meningiomas with focal chordoid histology ( $<50 \%$ chordoid histology) with available preoperative MR imaging examinations, including diffusion-weighted imaging, were identified. Qualitative imaging features and quantitative ADC values were compared between meningiomas with chordoid histology and 42 nonchordoid meningiomas (29 WHO grade I, eleven WHO grade II, and 2 WHO grade III).

RESULTS: The median ADC $\left(10^{-3} \mathrm{~mm}^{2} / \mathrm{s}\right)$ of meningiomas with chordoid histology was significantly higher than nonchordoid meningiomas (1.16 versus $0.92, P<.001$ ), as was the median normalized ADC (1.60 versus 1.19, $P<.001)$. In subgroup analysis, the median and normalized ADC values of chordoid meningiomas $(n=11)$ were significantly higher than those in meningiomas with focal chordoid histology $(n=10$, $P<.001$ and $P<.001$, respectively) or nonchordoid meningiomas ( $n=42, P<.001$ and $<0.001$, respectively). Median and normalized ADC values were not significantly different between the meningiomas with focal chordoid histology and nonchordoid meningiomas $(P=.816$ and .301 , respectively). Among the qualitative imaging features, only DWI signal intensity was significantly associated with meningiomas with chordoid histology diagnosis.

CONCLUSIONS: ADC values are higher in chordoid compared with nonchordoid meningiomas and may be used to discriminate the degree of chordoid histology in meningiomas. While qualitative MR imaging features do not strongly discriminate chordoid from nonchordoid meningiomas, DWI may allow preoperative identification of chordoid meningiomas.

ABBREVIATIONS: IQR = interquartile range; $\mathrm{MCH}=$ meningiomas with chordoid histology; $\mathrm{nADC}=$ normalized $\mathrm{ADC}$

hordoid meningiomas are a rare subtype of atypical, WHO grade II meningioma. ${ }^{1}$ By histopathology, chordoid menin- giomas are composed of spindled-to-epithelioid cells with eosinophilic cytoplasm arranged in chains and cords within a basophilic extracellular matrix. This myxoid stroma is rich in acidic mucin and stains with mucicarmine, periodic-acid-Schiff, and Alcian blue. Chordoid meningiomas are associated with higher rates of recurrence than benign WHO grade I meningiomas and have thus been designated as a grade II variant in the WHO classification, even in those examples lacking increased mitotic activity, brain invasion, or other atypical criteria. ${ }^{2-5}$ Thus, preoperative imaging identification of chordoid meningiomas could provide

\footnotetext{
Please address correspondence to Javier E. Villanueva-Meyer, MD, Department of Radiology and Biomedical Imaging, University of California San Francisco, 505 Parnassus, L352, San Francisco, CA 94143; e-mail: javier.villanueva-meyer@ucsf.edu - Indicates open access to non-subscribers at www.ajnr.org http://dx.doi.org/10.3174/ajnr.A5996
}

Received October 3, 2018; accepted after revision January 14, 2019.

From the Departments of Radiology and Biomedical Imaging (J.D.B., J.E.V.-M.), Radiation Oncology (W.C.C., J.S.P., J.H.H., D.R.R.), Pathology (D.A.S., C.-H.L.), and Neurocisco, California.

This work was supported by a grant from the Linda Wolfe Meningioma Research Fund to D.R.R. This publication was also supported by the National Center for Advancing Translational Sciences, National Institutes of Health, through University of TR001871.

Joe D. Baal, William C. Chen, David R. Raleigh, and Javier E, Villaneurva-Meyer contributed equally to this work.

The content of this work is solely the responsibility of the authors and does not necessarily represent the official views of the National Institutes of Health. 
valuable information to guide surgical planning, adjuvant therapy, and patient counseling.

Several studies have sought to define imaging features that can reliably distinguish low- and high-grade meningiomas. In that regard, features such as larger size and irregular shape are associated with a higher meningioma grade. ${ }^{6-9}$ Although sensitive, these associations have poor specificity and are thus unreliable for identifying atypical and anaplastic meningiomas. ${ }^{10}$ Investigators have addressed these limitations by analyzing advanced MR imaging with quantitative metrics, including diffusion, perfusion, and MR spectroscopy. ${ }^{11-17}$ To date, the most promising MR imaging feature for differentiating WHO grades in meningiomas is the apparent diffusion coefficient, a measure of the degree of diffusion of water molecules within tissue. ${ }^{11,12}$ Prior meningioma studies have revealed a significant correlation between whole-tumor ADC histogram metrics and tumor histology and corresponding WHO grade. ${ }^{18,19}$ However, only 1 study, which was limited to 4 patients, has specifically addressed the utility of ADC in identifying chordoid meningiomas on preoperative imaging. ${ }^{13}$

Given the relative paucity of imaging studies focused on chordoid meningiomas and the unmet need to identify high-grade meningiomas preoperatively, our study assessed whether qualitative or quantitative MR imaging features such as ADC can be used as preoperative MR imaging metrics to differentiate chordoid meningiomas from other meningioma subtypes. Our data revealed that ADC can delineate chordoid meningiomas from other meningioma histologies and can also identify the degree of chordoid histology within an individual meningioma.

\section{MATERIALS AND METHODS \\ Patient Population and Study Information}

We identified 24 patients who underwent resection for meningiomas with chordoid histology at our institution from 2000 to 2018. All resection specimens were re-reviewed by an expert neuropathologist (D.A.S.) to confirm the presence and extent of chordoid histology. Chordoid meningiomas ( $>50 \%$ chordoid histology, $n=11$ ) were considered separately from meningiomas with focal chordoid histology $(<50 \%$ chordoid histology, $n=$ 10 ), but for some analyses, these groups were combined as meningiomas with chordoid histology $(\mathrm{MCH}, n=21)$. A cohort of 42 patients with histologically confirmed nonchordoid meningioma were randomly selected from a consecutive institutional cohort of patients with meningiomas for comparison using a random number generator. Clinical variables, including patient age, sex, and meningioma grade, were extracted from the medical record. Only patients with preoperative MR imaging examinations with available diffusion-weighted imaging and complete clinical records were included in the analysis. Patients who underwent preoperative meningioma embolization before MR imaging were excluded. This retrospective study was approved by the institutional review board.

\section{MR Imaging Protocol}

MR imaging was performed within a week before surgical resection using a $1.5 \mathrm{~T}$ or $3 \mathrm{~T}$ MR imaging scanner. While the acquired MR imaging pulse sequences varied during the course of the study, at a minimum, the standard neuronavigation MR imaging protocol consisted of the following pulse sequences encompassing the entire brain: precontrast T1 and T2, T2 FLAIR, DWI (bvalues, 0 and $1000 \mathrm{~s} / \mathrm{mm}^{2}$ ), and gadolinium-enhanced 3D echospoiled gradient-echo T1-weighted images.

\section{MR Imaging Analysis}

A board-certified neuroradiologist (J.E.V.-M.) evaluated MR images for qualitative imaging features, including lesion location, focality, size, T1/T2/DWI signal intensity, the presence of a dural tail or CSF cleft sign, bony involvement, parenchymal edema, tumor location, dural venous sinus involvement, arterial narrowing, and the presence of sunburst vessels using a clinical PACS. Signal intensity was characterized relative to gray matter for T1- and T2-weighted imaging and relative to brain for DWI. ADC maps were exported to MIM Software (MIM Software, Cleveland, Ohio), in which meningiomas were contoured by a board-certified radiation oncologist with expertise in tumors of the central nervous system (D.R.R.). All contours were initially defined around the tumor on gadoliniumenhanced T1 images and individually verified to ensure that they accurately preserved meningioma borders. The contoured ROIs from gadolinium-enhanced T1 images were automatically coregistered with corresponding ADC maps to obtain whole-tumor mean $\mathrm{ADC}$ values. Control $\mathrm{ADC}$ values were measured from contralateral normal-appearing white matter. Normalized ADC (nADC) was calculated by dividing the meningioma $\mathrm{ADC}$ value by the respective control ADC value.

\section{Data Analysis}

All statistical analyses were performed in STATA 15.0 (StataCorp, College Station, Texas). Differences in overall ADC and nADC values between $\mathrm{MCH}$ and nonchordoid meningiomas were compared using Wilcoxon rank sum tests. Additional subgroup analysis was performed to investigate the difference in overall ADC and $\mathrm{nADC}$ values among chordoid meningiomas, meningiomas with focal chordoid histology, and nonchordoid meningiomas. Previously published cutoff values of ADC $\geq 1.4$ and nADC $\geq 1.9$ for identifying the chordoid histology were evaluated. ${ }^{13}$ Subsequently, a receiver operating characteristic analysis was performed to define optimal cutoff values for the ADC and $\mathrm{AADC}$. Univariate logistic and exact logistic regressions were performed to assess the predictive value of selected imaging features in preoperative chordoid meningioma diagnosis.

\section{RESULTS}

Of the 24 identified patients with $\mathrm{MCH}, 21$ met the inclusion criteria and were compared with 42 patients with nonchordoid meningiomas, which included 29 WHO grade I, eleven WHO grade II, and 2 WHO grade III meningiomas. Among the 10 meningiomas with focal chordoid features, the predominant meningioma histology included 6 WHO grade I, three WHO grade II, and WHO grade III. Approximately $70 \%$ of both MCHs and nonchordoid meningiomas were supratentorial. Similarly, both histologic groups predominantly presented as solitary lesions instead of as multifocal tumors. The proportions of $\mathrm{MCH}$ and nonchordoid meningiomas observed to have a dural tail, bony involvement, a cystic component, CSF cleft sign, dural venous sinus involvement, arterial narrowing, sunburst vessels, irregular mar- 
Univariate analysis of qualitative MR imaging features for chordoid meningioma

\begin{tabular}{|c|c|c|}
\hline \multirow[b]{2}{*}{ Predictor Variables } & \multicolumn{2}{|c|}{$\begin{array}{l}\text { Chordoid Meningioma } \\
\qquad(N=21)\end{array}$} \\
\hline & OR $(95 \% \mathrm{CI})$ & $P$ Value \\
\hline Supratentorial location & $1.43(0.43-4.75)$ & .555 \\
\hline Multifocality & $4.06(0.87-19.04)$ & .075 \\
\hline $\mathrm{T}$ hyperintensity & $1.34(0.46-3.91)$ & .590 \\
\hline $\mathrm{T} T+$ marked $\mathrm{CE}^{\mathrm{a}}$ & 6.49 (0.93 to +inf) & .061 \\
\hline T2 hyperintensity & $2.91(0.90-9.40)$ & .074 \\
\hline ADC hyperintensity & $4.29(1.31-13.98)$ & $.016^{\mathrm{b}}$ \\
\hline Presence of dural tail ${ }^{a}$ & $0.50(0-19.50)$ & .667 \\
\hline Bony involvement & $3.10(0.86-8.06)$ & .090 \\
\hline Cystic/necrotic change & $2.31(0.59-9.11)$ & .231 \\
\hline Sunburst vessels & $1.00(0.29-3.42)$ & 1.000 \\
\hline Venous involvement ${ }^{\mathrm{a}}$ & $1.57(0.21-10.40)$ & .859 \\
\hline Arterial narrowing & $0.48(0.05-4.54)$ & .518 \\
\hline CSF cleft & $0.44(0.14-1.40)$ & .165 \\
\hline Parenchymal edema & $1.10(0.38-3.24)$ & .856 \\
\hline Irregular margins ${ }^{a}$ & $6.05(0.88-69.7)$ & .072 \\
\hline
\end{tabular}

Note:-CE indicates contrast enhancement; inf, infinity.

axact logistic regression.

Significant. gins, and parenchymal edema on imaging were $95 \%$ versus $100 \%$, $52 \%$ versus $26 \%, 24 \%$ versus $12 \%, 62 \%$ versus $79 \%, 14 \%$ versus $10 \%, 5 \%$ versus $9.5 \%, 24 \%$ versus $24 \%, 24 \%$ versus $5 \%$, and $62 \%$ versus $60 \%$, respectively. T1 signal hyperintensity was observed in approximately $62 \%$ of $\mathrm{MCH}$ and $55 \%$ of nonchordoid meningiomas. T2 signal hyperintensity was observed in $76 \%$ of $\mathrm{MCH}$ and $52 \%$ of nonchordoid meningiomas. Marked T1 contrast enhancement was observed in $100 \%$ of $\mathrm{MCH}$ and $81 \%$ of nonchordoid meningiomas. Qualitative facilitated diffusion (hyperintensity on ADC with hypointensity on DWI) was seen in $48 \%$ of $\mathrm{MCH}$ and $18 \%$ of nonchordoid meningiomas. On univariate logistic and exact logistic regressions, only ADC hyperintensity was found to be significantly associated with $\mathrm{MCH}$ diagnosis $(P=.016)$ (Table).

Intratumoral $\mathrm{ADC}$ values $\left(10^{-3} \mathrm{~mm}^{2} / \mathrm{s}\right)$ in $\mathrm{MCH}$ ranged from 0.75 to 1.86 , and the corresponding $\mathrm{nADC}$ values ranged from 1.11 to 2.49 (Figs 1 and 2). In nonchordoid meningiomas, the intratumoral $\mathrm{ADC}$ values ranged from 0.79 to 1.09 , and the corresponding $\mathrm{nADC}$ values ranged from 1.01 to 1.42 (Fig 3). The median ADC of all $\mathrm{MCHs}(1.16$; interquartile range $[\mathrm{IQR}]=0.60)$ was significantly higher than that in nonchordoid meningiomas $(0.92, \mathrm{IQR}=0.12$,
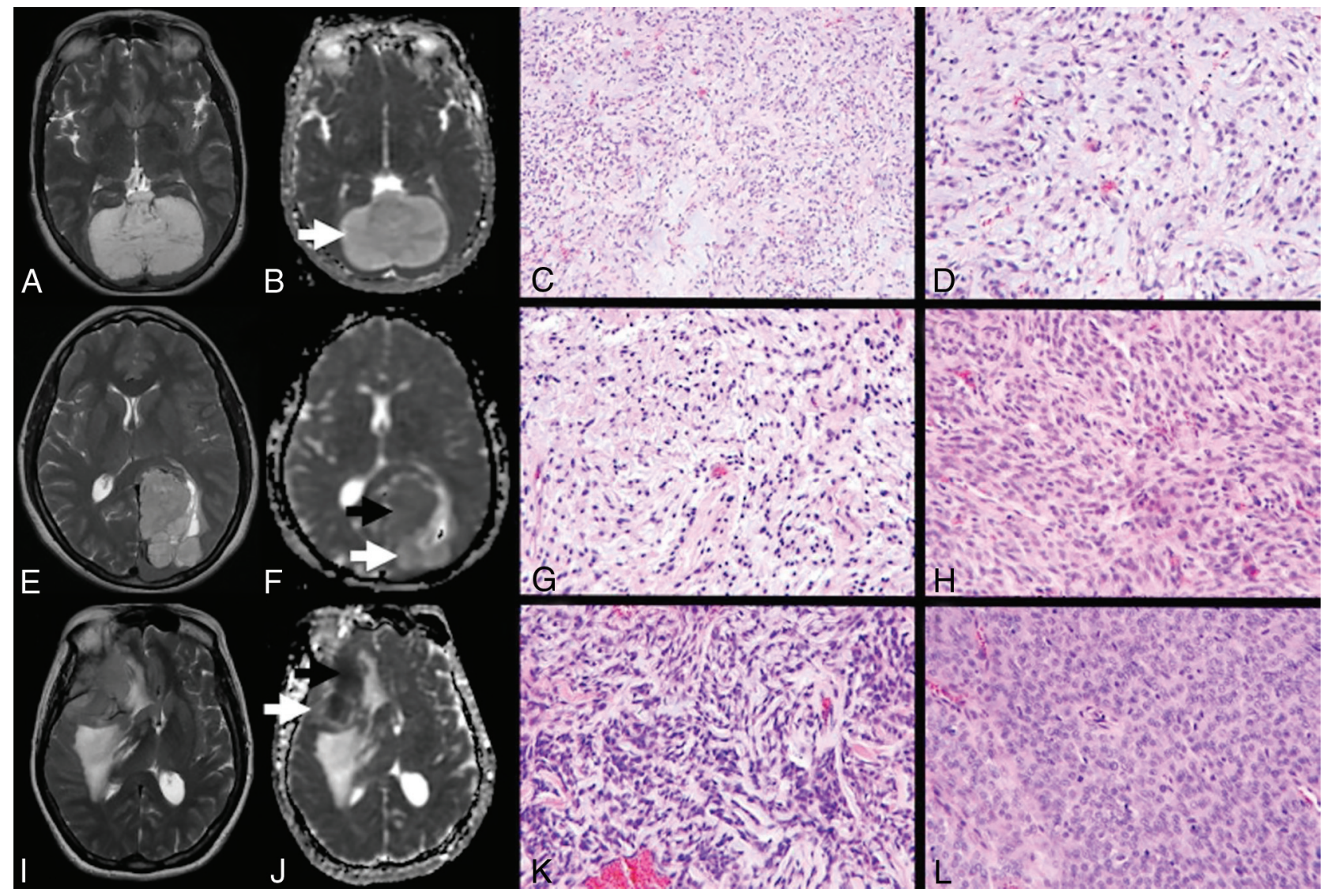

FIG 1. Meningiomas with chordoid histology. $A-D$, Chordoid meningioma. Axial T2-weighted image $(A)$ demonstrates a T2 hyperintense falcotentorial meningioma with facilitated diffusion on the ADC map (B, white arrow). Hematoxylin-eosin (H\&E) stained sections at 20X (C) and 40x (D) magnification demonstrate chains and clusters of epithelioid cells in a basophilic myxoid stroma characteristic of chordoid meningioma. $E-H$, Meningiomas with focal chordoid features. Axial T2-weighted image (E) demonstrates a T2-hyperintense left posterior parasagittal meningioma. The corresponding ADC map $(F)$ demonstrates a dominant area of signal isointensity (black arrow) with focal facilitated diffusion (white arrow). H\&E-stained sections at $40 \times$ magnification demonstrate regions of chordoid $(G)$ and conventional meningothelial $(H)$ histology. $I-L$, Anaplastic meningioma with focal chordoid features. Axial T2-weighted image $(I)$ demonstrates a heterogeneous right sphenoid wing meningioma. The corresponding ADC map $(U)$ demonstrates regions of reduced diffusion (black arrow), suggesting increased tumoral cellularity, with a small focus of facilitated diffusion (white arrow). H\&E-stained sections at 40X magnification demonstrate focal regions of chordoid histology $(K)$, with a predominant component of anaplastic meningioma lacking chordoid features $(L)$. 


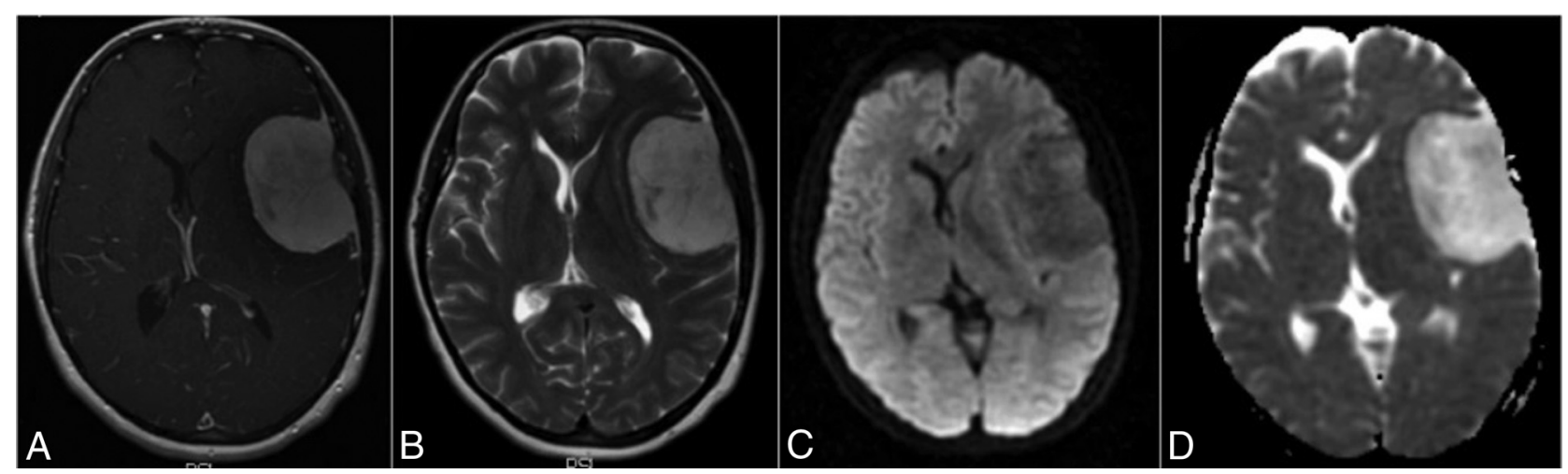

FIG 2. Left frontal chordoid meningioma. A, Postcontrast Tl-weighted image of a $5.5 \times 3.6 \mathrm{~cm}$ chordoid meningioma shows clear enhancement with areas of nonenhancement. $B, T 2$-weighted image shows moderate-to-hyperintense signal. $C$, DWI shows hypointense signal. $D, A$ corresponding ADC map demonstrates marked hyperintense signals from the tumor with resulting $A D C$ and $n A D C$ values of $1.84 \times 10^{-3} \mathrm{~mm}^{2} / \mathrm{s}$ and 2.36 , respectively.

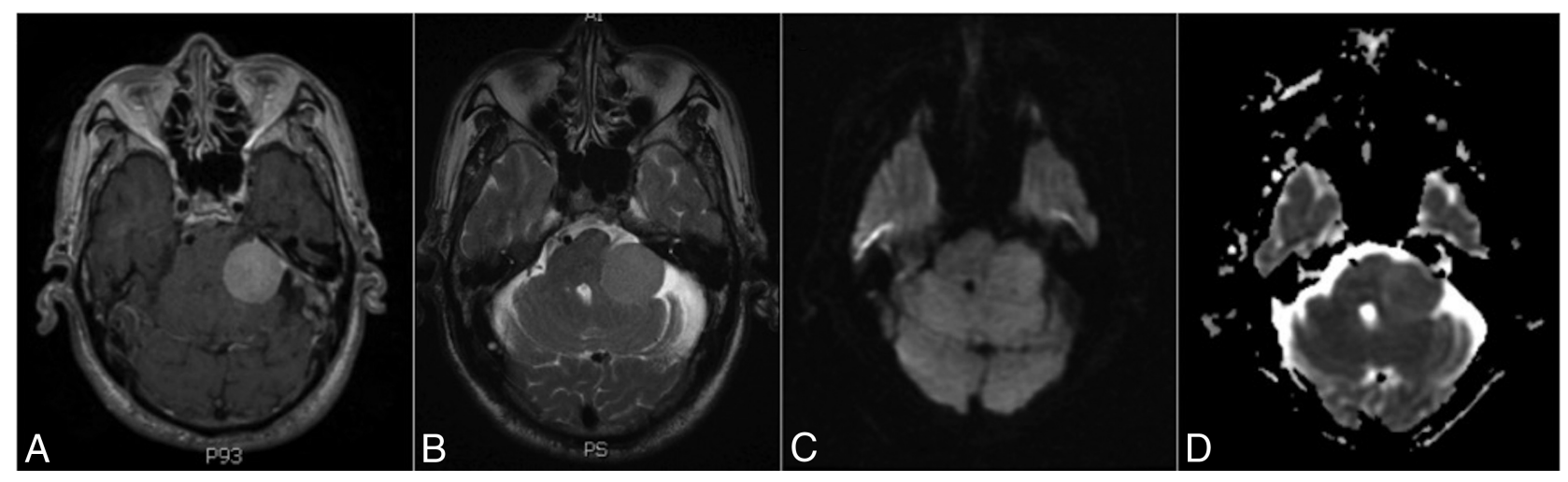

FIG 3. Left cerebellopontine angle nonchordoid meningioma, WHO grade I. A, Postcontrast T1-weighted image shows homogeneous enhancement in the $3.0 \times 2.8 \mathrm{~cm}$ nonchordoid meningioma. $B$, T2-weighted image shows moderately intense signals. $C$, DWI shows isointense signals. $D$, A corresponding ADC map demonstrates isointense signal with resulting ADC and nADC values of $0.94 \times 10^{-3} \mathrm{~mm} \mathrm{~m}^{2} / \mathrm{s}$ and 1.26 , respectively.

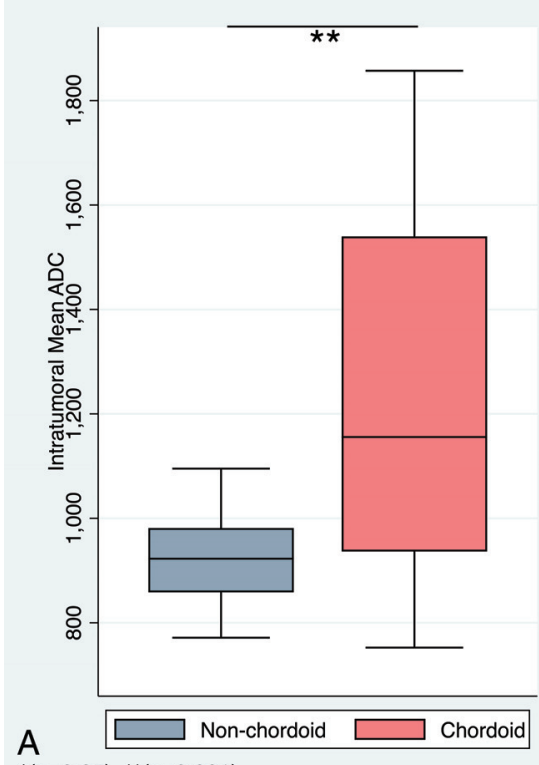

${ }^{*}(p<0.05),{ }^{* *}(p<0.001)$

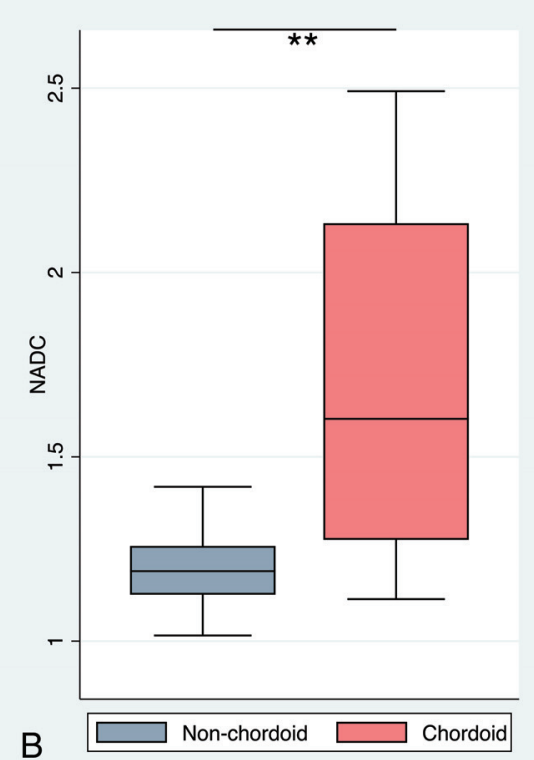

B

FIG 4. Distribution of $A D C$ and $n A D C$ values between chordoid and nonchordoid meningiomas. Boxplots of $A D C\left(\times 10^{-6} \mathrm{~mm}^{2} / \mathrm{s}\right)(A)$ and $\mathrm{nADC}(B)$ values of nonchordoid (gray) and chordoid meningiomas (red).
$P<.001$ ) (Fig $4 A$ ). Similarly, the median $\mathrm{nADC}$ of MCHs $(1.60, \mathrm{IQR}=0.86)$ was significantly higher than that of the nonchordoid meningiomas $(1.19, \mathrm{IQR}=$ $0.13, P<.001)$ (Fig $4 B)$. Subgroup analysis revealed a significant difference in the median ADC between chordoid meningioma $(1.54$, IQR $=0.24, n=11)$ and meningiomas with focal chordoid features (0.93, IQR $=0.07, n=10, P<.001)($ Fig $5 A)$. The median nADC of chordoid meningioma $(2.13, \mathrm{IQR}=0.41)$ was also significantly higher than that in meningiomas with focal chordoid features (1.30, $\mathrm{IQR}=0.20, P<.001)$ (Fig 5B). Consistently, chordoid meningiomas had significantly higher median ADC compared with nonchordoid meningiomas (0.92, $P<.001$ ), while the median ADC in meningiomas with focal chordoid features was not significantly different from that in nonchordoid meningiomas $(P=.816)$ (Fig 6A). Furthermore, the median nADC values were significantly higher in chor- 


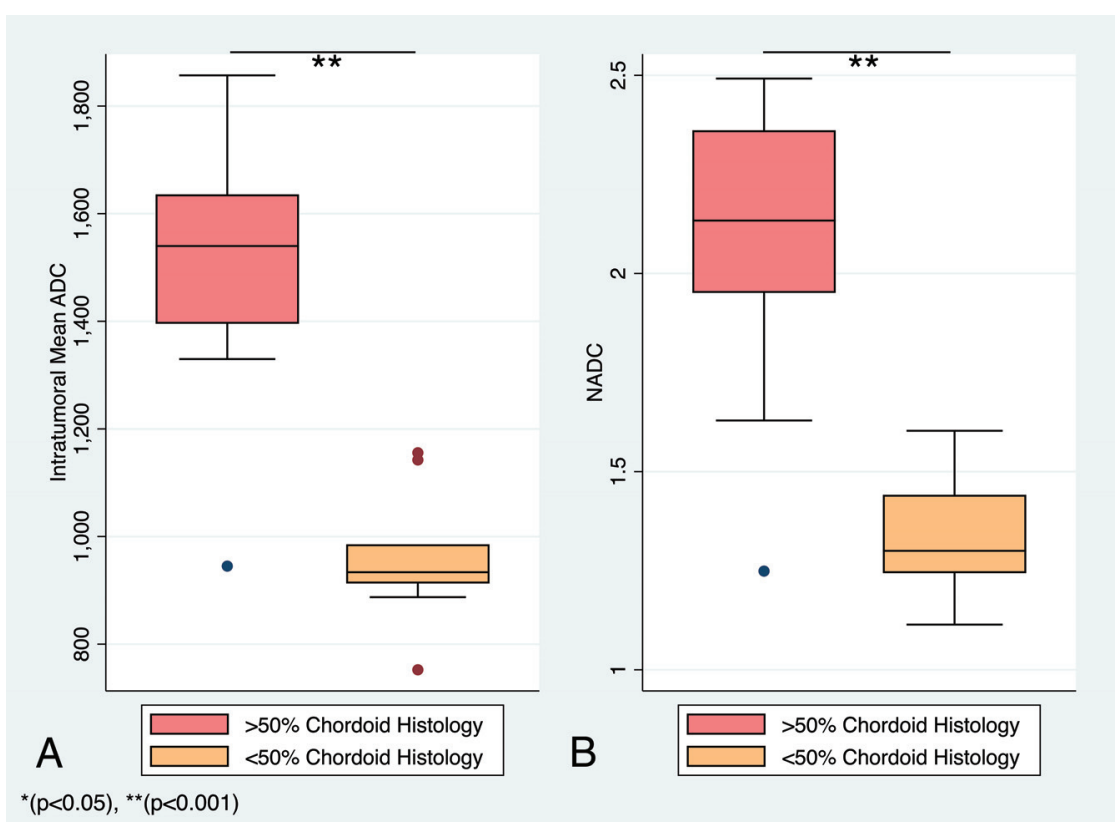

FIG 5. Distribution of $A D C$ and $n A D C$ values by an intratumoral proportion of chordoid histology. Boxplots of $A D C\left(\times 10^{-6} \mathrm{~mm}^{2} / \mathrm{s}\right)(A)$ and $\mathrm{nADC}(B)$ values of chordoid meningiomas (red) and meningiomas with focal chordoid features (orange).

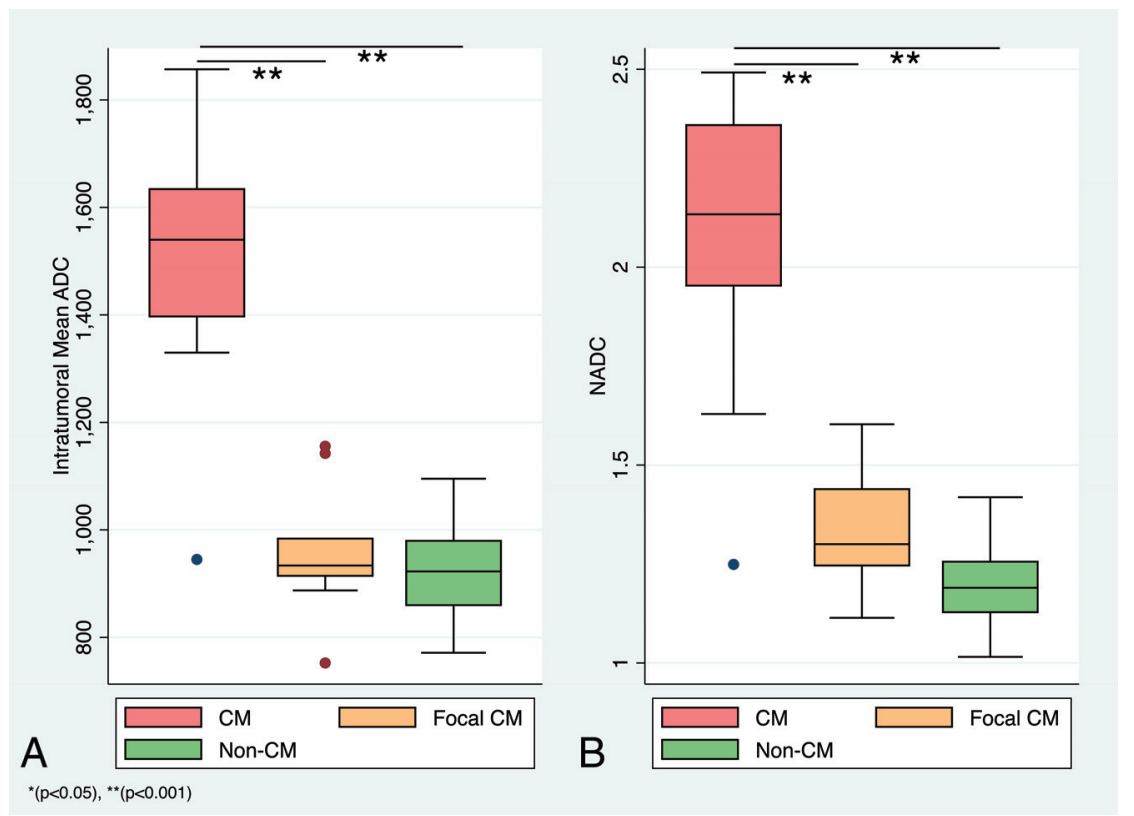

FIG 6. $A D C$ and $n A D C$ values among chordoid meningioma, meningiomas with focal chordoid histology, and nonchordoid meningiomas. Boxplots of $A D C\left(\times 10^{-6} \mathrm{~mm}^{2} / \mathrm{s}\right)(A)$ and $\mathrm{nADC}(B)$ values of chordoid meningiomas (red), meningiomas with focal chordoid features (orange), and nonchordoid meningiomas (green).

doid meningiomas versus nonchordoid meningiomas $(1.19, P<$ .001) (Fig 6B). There was no statistically significant difference in median $\mathrm{nADC}$ values between meningiomas with focal chordoid features and nonchordoid meningiomas $(P=.301)$.

The median $\operatorname{ADC}(1.16, \mathrm{IQR}=0.60)$ and $\mathrm{nADC}(1.60, \mathrm{IQR}=$ $0.86)$ of $\mathrm{MCH}$ WHO grade II tumors were significantly higher than the median $\mathrm{ADC}(0.89, \mathrm{IQR}=0.22, P=.005)$ and $\mathrm{nADC}$ (1.21, IQR $=0.15, P=.001)$ of nonchordoid WHO grade II meningiomas. Similarly, the median ADC and nADC of MCHs were significantly elevated compared with the median $\mathrm{ADC}(0.86, \mathrm{IQR}=$ $0.04, P=.038)$ and $\mathrm{nADC}(1.15, \mathrm{IQR}=$ $0.14, P=.038)$ of WHO grade III meningiomas.

The overall diagnostic accuracy of the previously defined cutoff value of $\mathrm{ADC} \geq 1.39 \times 10^{-3} \mathrm{~mm}^{2} / \mathrm{s}$ to identify chordoid meningioma on preoperative imaging was $96.8 \%$, with a sensitivity and specificity of $96.3 \%$ and $100 \%$, respectively. ${ }^{13}$ The overall diagnostic accuracy of the previously defined cutoff value of $\mathrm{nADC} \geq 1.9$ was $96.8 \%$, with a sensitivity and specificity of $96.3 \%$ and $100 \%$, respectively. On receiver operating characteristic analysis, cutoff values at $\mathrm{ADC} \geq 1.33 \times 10^{-3} \mathrm{~mm}^{2} / \mathrm{s}$ and $\mathrm{nADC} \geq 1.63$ were identified with resulting identical overall accuracy, sensitivity, and specificity of $96.8 \%, 96.3 \%$, and $100 \%$, respectively.

\section{DISCUSSION}

We found that both ADC and nADC values are significantly higher in MCHs compared with nonchordoid meningiomas. Moreover, our data reveal that chordoid meningiomas have significantly higher $\mathrm{ADC}$ and $\mathrm{nADC}$ values than either meningiomas with focal chordoid features or nonchordoid meningiomas. This finding remains true at a qualitative level, at which high ADC signal intensity and corresponding low DWI signal intensity are enriched in the $\mathrm{MCH}$ group.

Typically, WHO grade II and III meningiomas have greater intratumoral cellularity, increased tissue density, and decreased extracellular space, all of which are thought to contribute to decreased free water diffusion. ${ }^{11,12}$ Despite their WHO grade II classification, chordoid meningiomas have been found to have elevated ADC values. ${ }^{13}$ Increased water diffusivity within chordoid meningiomas is believed to be linked to an extracellular network composed of hyaluronic acid and chondroitin sulfate-rich mucoid matrix, which can be observed on microscopic evaluation and is unique to the chordoid subtype of meningioma. ${ }^{4}$ Most interesting, several studies have found associations between elevated ADC values and other types of tumors that are enriched with myxoid stroma, such as myxoid softtissue tumors, chordomas, and chondrosarcomas. ${ }^{14,15}$ Overall, our findings coincide with those reported in the only other investigation of DWI in chordoid meningioma. ${ }^{13}$ The overall mean ADC and $\mathrm{nADC}$ values for $\mathrm{MCH}$ in our study were lower compared with those found in the previous study $\left(1.62 \pm 0.33 \times 10^{-3} \mathrm{~mm}^{2} / \mathrm{s}\right.$ and 
$2.22 \pm 0.47$, respectively). However, the mean ADC and nADC values of the chordoid meningioma group in our study are similar to the mean values of the previous study. Given that ADC values were studied in only 4 chordoid meningiomas in the previous report, it is possible that all the chordoid meningiomas in that sample were of chordoid-predominant histology. Nonetheless, their previously defined $\mathrm{ADC}$ and $\mathrm{nADC}$ cutoff values of $1.39 \times 10^{-3} \mathrm{~mm}^{2} / \mathrm{s}$ and 1.93 had the same diagnostic accuracy as the defined cutoff values of our study with identically high sensitivity and specificity.

While there have been prior clinicopathologic studies on the proportion of chordoid meningioma histology, ${ }^{4,5}$ our study is the first to investigate imaging features associated with chordoid meningiomas on the basis of the relative proportion of chordoid histology. Mean $\mathrm{ADC}$ and $\mathrm{nADC}$ values were highest in chordoid meningiomas, consistent with the theorized greater water diffusivity in meningiomas with $>50 \%$ chordoid composition. Indeed, chordoid meningiomas had $\mathrm{ADC}$ and $\mathrm{nADC}$ values that were significantly increased compared with both meningiomas with focal chordoid features and nonchordoid meningiomas. In contrast, the mean ADC and nADC values were not significantly different between meningiomas with focal chordoid histology and nonchordoid meningiomas. With $<50 \%$ chordoid histology, the degree of water diffusivity in meningiomas with focal chordoid histology seems to be principally driven by the prevailing histology within the tumor. These findings highlight not only the heterogeneity of meningioma histology but also the potential limitation of ADC in identifying meningiomas with $<50 \%$ chordoid histology.

Qualitative assessment of ADC hyperintensity proved useful in distinguishing $\mathrm{MCH}$ from nonchordoid meningiomas. ADC hyperintensity with corresponding DWI hypointensity was observed in a greater proportion of MCHs than in nonchordoid meningiomas and was significantly associated with approximately 4 times the odds of chordoid meningioma diagnosis. Our findings support the utility of qualitative assessment of DWI sequences in identifying MCHs, which is more feasible in routine clinical practice compared with quantitative $\mathrm{ADC}$ analysis. Otherwise, most qualitative imaging features were not significantly associated with $\mathrm{MCH}$ diagnosis. Of note, while chordoid meningioma histology is rich with mucoid matrix that is typically associated with increased T2 signal, especially in other tumors such as chordomas or chondrosarcomas, ${ }^{4,15} \mathrm{~T} 2$ signal hyperintensity did not achieve statistical significance in this study. Overall, the scarcity of statistically significant qualitative imaging features associated with chordoid histology in meningioma diagnosis further highlights the importance of quantitative metrics like DWI in preoperative meningioma diagnosis.

There are several limitations to our study. As a retrospective study, our data were limited not only by the availability of suitable preoperative imaging including DWI but also by the rarity of chordoid meningioma. Despite the small number of cases in our study, it is the largest study of its kind to date. Another limitation may be in the measurement of ADC values. While our study did not use 2 independent extractions of ADC values to assess interobserver reliability, all of the contours were individually assessed to guarantee precise adherence to meningioma borders while avoiding areas of signal loss that could alter quantifications. Consistently, all contours were derived from postcontrast imaging and further cross-referenced with $\mathrm{T} 1 / \mathrm{T} 2$ sequences for accurate coverage of the meningioma area. Last, because contours of the whole tumor were used, there is greater reproducibility as opposed to simply using a single-slice ROI within the tumor. Using whole-tumor data allows more accurate representation of the total diffusion profile that may vary within the microarchitecture of a single tumor, as demonstrated by prior ADC histogram analyses on meningiomas. ${ }^{18}$ Most interesting, using whole-tumor measurements and histogram analysis may also better quantify morphologic imaging metrics such as signal intensity for predicting tumor histology, as shown by some studies. ${ }^{20,21}$

\section{CONCLUSIONS}

Our study demonstrates statistically significant elevations of ADC and $\mathrm{nADC}$ values in chordoid meningiomas compared with meningiomas with focal chordoid features and nonchordoid meningiomas. Our study also reveals that quantitative ADC may have additional utility in classifying the proportion of intratumoral chordoid histology on preoperative imaging. Cutoff values of $\mathrm{ADC} \geq 1.33$ $\times 10^{-3} \mathrm{~mm}^{2} / \mathrm{s}$ and $\mathrm{nADC} \geq 1.63$ can be used to help identify potential chordoid meningiomas on preoperative imaging with considerable diagnostic accuracy. Preoperative identification of chordoid meningiomas could provide valuable information to guide subsequent surgical planning, adjuvant therapy, and patient counseling.

Disclosures: Joe D. Baal—RELATED: Grant: National Institutes of Health TL1 TR001871.* *Money paid to the institution.

\section{REFERENCES}

1. Louis DN, Perry A, Reifenberger G, et al. The 2016 World Health Organization classification of tumors of the central nervous system: a summary. Acta Neuropathol 2016;131:803-20

2. Kepes JJ, Chen WY, Connors MH, et al. “Chordoid” meningeal tumors in young individuals with peritumoral lymphoplasmacellular infiltrates causing systemic manifestations of the Castleman syndrome: a report of seven cases. Cancer 1988;62:391-406 CrossRef Medline

3. Yang Y, Li D, Cao XY, et al. Clinical features, treatment, and prognostic factors of chordoid meningioma: radiological and pathological features in $\mathbf{6 0}$ cases of chordoid meningioma. World Neurosurg 2016;93:198-207 CrossRef Medline

4. Couce ME, Aker FV, Scheithauer BW. Chordoid meningioma: a clinicopathologic study of $\mathbf{4 2}$ cases. Am J Surg Pathol 2000;24:899905 CrossRef Medline

5. Epari S, Sharma MC, Sarkar C, et al. Chordoid meningioma, an uncommon variant of meningioma: a clinicopathologic study of 12 cases. J Neurooncol 2006;78:263-69 CrossRef Medline

6. Carpeggiani P, Crisi G, Trevisan C. MRI of intracranial meningiomas: correlations with histology and physical consistency. Neuroradiology 1993;35:532-36 CrossRef Medline

7. Zimmerman RD, Fleming CA, Saint-louis LA, et al. Magnetic resonance imaging of meningiomas. AJNR Am J Neuroradiol 1985;6: 149-57 Medline

8. Coroller TP, Bi WL, Huynh E, et al. Radiographic prediction of meningioma grade by semantic and radiomic features. PLoS One 2017; 12:e0187908 CrossRef Medline

9. Nakasu S, Nakasu Y, Nakajima M, et al. Preoperative identification of meningiomas that are highly likely to recur. J Neurosurg 1999;90: 455-62 Medline

10. Watts J, Box G, Galvin A, et al. Magnetic resonance imaging of meningiomas: a pictorial review. Insights Imaging 2014;5:113-22 CrossRef Medline

11. Filippi CG, Edgar MA, Uluğ AM, et al. Appearance of meningiomas on diffusion-weighted images: correlating diffusion constants with histopathologic findings. AJNR Am J Neuroradiol 2001;22:65-72 Medline

12. Nagar VA, Ye JR, Ng WH, et al. Diffusion-weighted MR imaging: 
diagnosing atypical or malignant meningiomas and detecting tumor dedifferentiation. AJNR Am J Neuroradiol 2008;29:1147-52 CrossRef Medline

13. Pond JB, Morgan TG, Hatanpaa KJ, et al. Chordoid meningioma: differentiating a rare World Health Organization grade II tumor from other meningioma histologic subtypes using MRI. AJNR Am J Neuroradiol 2015;36:1253-58 CrossRef Medline

14. Nagata S, Nishimura H, Uchida M, et al. Diffusion-weighted imaging of soft tissue tumors: usefulness of the apparent diffusion coefficient for differential diagnosis. Radiat Med 2008;26:287-95 CrossRef Medline

15. Yeom KW, Lober RM, Mobley BC, et al. Diffusion-weighted MRI: distinction of skull base chordoma from chondrosarcoma. AJNR Am J Neuroradiol 2013;34:1056-61, S1 CrossRef Medline

16. Yang S, Law M, Zagzag D, et al. Dynamic contrast-enhanced perfusion MR imaging measurements of endothelial permeability: differentiation between atypical and typical meningiomas. AJNR Am J Neuroradiol 2003;24:1554-59 Medline

17. Zhang H, Rödiger LA, Shen T, et al. Perfusion MR imaging for dif- ferentiation of benign and malignant meningiomas. Neuroradiology 2008;50:525-30 CrossRef Medline

18. Gihr GA, Horvath-Rizea D, Garnov N, et al. Diffusion profiling via a histogram approach distinguishes low-grade from high-grade meningiomas, can reflect the respective proliferative potential and progesterone receptor status. Mol Imaging Biol 2018;20:632-40 CrossRef Medline

19. Schob S, Surov A, Wienke A, et al. Correlation between aquaporin 4 expression and different DWI parameters in grade I meningioma. Mol Imaging Biol 2017;19:138-42 CrossRef Medline

20. Schob S, Frydrychowicz C, Gawlitza M, et al. Signal intensities in preoperative MRI do not reflect proliferative activity in meningioma. Transl Oncol 2016;9:274-79 CrossRef Medline

21. Gihr GA, Horvath-Rizea D, Kohlhof-Meinecke P, et al. Histogram profiling of postcontrast T1-weighted MRI gives valuable insights into tumor biology and enables prediction of growth kinetics and prognosis in meningiomas. Transl Oncol 2018;11:957-61 CrossRef Medline 